\title{
Characterization of SINR Region for Interfering Links with Constrained Power
}

\author{
Hajar Mahdavi-Doost, Masoud Ebrahimi, and Amir K. Khandani
}

\begin{abstract}
In this paper, a communication system including $n$ interfering additive white Gaussian noise (AWGN) links is considered. Each transmitter uses a Gaussian codebook and each receiver only decodes the data of the corresponding transmitter. For the case that the transmit powers are subject to arbitrary linear constraints, a closed-form expression for the boundary points of the signal-to-interference-plusnoise-ratio (SINR) region is obtained. Moreover, when the channels are time-varying and the average powers are constrained, the zero-outage SINR region of the system is derived. In addition, a scenario where the demanded SINR of the users is out of the SINR region is considered. A common approach is to remove a subset of the users such that the demanded SINR can be provided for the remaining users; the removed users are serviced in a later time slot. With the aim of maximizing the number of serviced users in each time slot, a sub-optimal algorithm is developed, which outperforms the other alternatives.
\end{abstract}

\section{Index Terms}

SINR region, rate region, maximum achievable SINR, time-varying channel, zero-outage SINR region, user removal.

\section{INTRODUCTION}

A collection of transmitter-receiver pairs operating in a shared medium constitute a system of interfering communication links. Practical examples of such configurations include cellular

This work is financially supported by Nortel Networks and by matching funds from the federal government of Canada (NSERC) and province of Ontario (OCE).

The authors are affiliated with the Coding and Signal Transmission Laboratory, Electrical and Computer Engineering Department, University of Waterloo, Waterloo, ON, N2L 3G1, Canada, Tel: 519-885-1211 ext. 35324, Fax: 519-888-4338, Emails: \{hajar, masoud, khandani\}@cst.uwaterloo.ca.

This material was presented in part at the 10th Canadian Workshop on Information Theory (CWIT), Edmonton, AB, Canada, June 2007 and IEEE International Symposium on Information Theory (ISIT), Nice, France, June 2007. 
networks, code division multiple access (CDMA), and digital subscriber line (DSL) systems. Due to the complexity of such structures, usually some simplifying assumptions are used in the design and analysis of such configurations. A widely used assumption is to consider Gaussian signal transmission and treat the interference as AWGN. In this case, the Shannon's capacity formula for AWGN channels is in effect. We follow this assumption throughout this paper.

The systems including interfering links have been the subject of research for many years. Based on the network structure, these systems have been approached in different ways, e.g. by power control [1], feasible region description [2]-[7], bandwidth allocation [8], transmission scheduling [9], routing [10], base station selection [11], etc. In this work, we are mainly concerned with characterizing the feasible SINR region of such systems when the transmission powers can take any value within some linear constraints. According to our assumption, there is a one-to-one logarithmic relation between the rate and the SINR of the users. Hence, by describing the feasible SINR region, we are describing the feasible rate region, as well.

In general, a feasible region for interfering links is defined based on a network parameter such as SINR, rate, capacity, processing gain, etc. In [2], it is shown that the feasible processing gain region when the power is unbounded is convex. Note that for a constant bandwidth, the processing gain is inversely proportional to the rate. Some topological properties of the mentioned feasible region are investigated in [3] for the cases when there are constraints on the power of individual users and when there is no constraint on the power. It is shown that the boundary of the capacity region with one user's power fixed and the rest unbounded is a shift of the boundary of some capacity region with modified parameters, but unlimited power. However, this result is not in a closed form and cannot be extended to the other forms of power constraints.

The study of the feasible SINR region shows that it is not convex in general [4], [5]. In [6], it is shown that in the case of unlimited power, the feasible SINR region is log-convex. The authors in [2] also consider a CDMA system without power constraints, and show that the feasible inverse-SINR region is convex. In [4], it is proved that for a given quality of service (QoS) parameter, the corresponding feasible region is convex, if the SINR is a log-convex function of that QoS parameter. Reference [7] shows that under a total power constraint, the infeasible SINR region is not convex.

In this paper, we consider the feasible SINR region and describe it by analytically obtaining its boundary points. Our approach is to find the farthest point of the feasible SINR region from origin 
in a given direction. This makes the problem of SINR region characterization closely related to the problem of maximizing the minimum SINR. There has been some effort to evaluate the maximum achievable SINR in a system of interfering links. In [12], the maximum achievable SINR of a system with no constraint on the power is expressed in terms of the Perron-Frobenius (PF) eigenvalue of a non-negative matrix. Then, this expression is utilized to develop an SINRbalancing scheme for satellite networks. This formulation for the maximum achievable SINR is deployed in many other wireless communication applications such as [13], [14].

It is not a straightforward task to generalize the elegant result of [12] for the realistic scenario when the transmit powers are constrained. As a result, [12] remained as the only work of this kind for many years. Recently, for a special case when the total power is constrained, the maximum achievable SINR is obtained based on the PF-eigenvalue of an $(n+1) \times(n+1)$ primitive non-negative matrix [15]. In this paper, we adopt an approach that enables us to obtain closed form expressions for the maximum achievable SINR in systems of interfering links with power constraints. Our approach is general in the sense that it can be applied to systems with various number of arbitrary linear power constraints. Moreover, this result yields a closed-form solution for the SINR region of the systems with power constraints, in terms of the PF-eigenvalue of an $n \times n$ irreducible matrix.

Later, we apply the mentioned approach to a time-varying system where the channel gains are selected from a finite set with certain probabilities, and the average power of users are subject to some linear constraints. For this system, we obtain the SINR region which is achievable regardless of the channel realizations. This region is known as the zero-outage SINR region [16], [17, Page 111-112]. In [16], the optimal zero-outage SINR for a point-to-point channel is obtained. Our result implements the same concept when we have $n$ interfering links with finite number of channel realizations.

It is likely in practical communication systems that the required SINR of the users is a point out of the feasible SINR region. In this case, one possible solution is to remove some of the users such that the required SINR of the remaining users falls in the feasible region of those users; the removed users are serviced in the subsequent time slots when their channel is in a better condition. This approach is inherent to any opportunistic scheduling problem [9]. With this approach, it is desirable to find a feasible subset of users (i.e., a subset of users which satisfy the required SINR) with maximum cardinality [18]. This problem is claimed to be NP- 
complete [19]. In the literature, some heuristic algorithms are presented for this problem. In [13], a stepwise removal algorithm (SRA) has been proposed for the case that the transmit power is unbounded. In [20], another algorithm named as stepwise-maximum-interference-removalalgorithm (SMIRA) is proposed, and it is shown that this algorithm outperforms SRA. For the systems with constraint on the power of the individual transmitters, an algorithm known as gradually-removal-distributed-constrained-power-control (GRX-DCPC) is proposed in [19]. This algorithm is presented in the forms of centralized, distributed, restricted, and non-restricted user selection. In the restricted algorithm known as GRR-DCPC, the user to be removed is selected from the users attaining the maximum power in the power updating procedure. Whereas, in the non-restricted algorithm (GRN-DCPC), the user to be removed is selected from all active users. The simulation results show that GRN-DCPC (centralized non-restricted algorithm) outperforms other mentioned schemes in [19].

In this paper, we exploit the obtained results on the maximum achievable SINR, to develop a suboptimal algorithm which maximizes the number of active users satisfying a required SINR and some power constraints. The algorithm is flexible for any linear power constraint. We consider two sorts of constraints on power: (i) individual constraints on the power of all users, and (ii) constraint on the total power of all users. Simulation results show that the proposed algorithm outperforms the alternative schemes in both cases in terms of the number of active users.

The rest of the paper is organized as follows: In Section II, the system model and problem formulation are presented. The SINR region for this system is characterized in Section III. Section IV is dedicated to the systems with time-varying channels. The removal algorithms are proposed in Section V. Finally, the paper is concluded in Section VI.

Notation: All boldface letters indicate column vectors (lower case) or matrices (upper case). $x_{i j}$ and $\mathbf{x}_{i}$ represent the entry $(i, j)$ and column $i$ of the matrix $\mathbf{X}$, respectively. A matrix $\mathbf{X}_{n \times m}$ is called non-negative and denoted by $\mathbf{X} \geq \mathbf{0}$ if $x_{i j} \geq 0 \quad \forall i \in\{1, \ldots, n\}$ and $j \in\{1, \ldots, m\}$. Also, $\mathbf{X} \geq \mathbf{Y}$ is equivalent to $\mathbf{X}-\mathbf{Y} \geq \mathbf{0}$, where $\mathbf{X}, \mathbf{Y}$ and $\mathbf{0}$ are non-negative matrices of compatible dimensions [21]. $\operatorname{det}(\mathbf{X}), \operatorname{Tr}(\mathbf{X}), \mathbf{X}^{\prime}$, and $|\mathbf{X}|$ denote the determinant, the trace, the transpose, and the Euclidean norm of the matrix $\mathbf{X}$, respectively. $\mathbf{I}$ is an identity matrix with compatible size. $\otimes$ represents the Kronecker product operator. $\operatorname{diag}(\mathbf{x})$ is a diagonal matrix whose main diagonal is $\mathbf{x}$. We define the reciprocal of a polynomial $\mathrm{q}(x)$ of degree $m$ as $x^{m} \mathrm{q}\left(\frac{1}{x}\right)$. For 
a matrix $\mathbf{X}$, a vector $\mathbf{y}$, and a set of indices $\mathcal{S}$, the matrix $\mathbf{Z}=\psi(\mathbf{X}, \mathbf{y}, \mathcal{S})$ is defined as

$$
\mathbf{z}_{j}= \begin{cases}\mathbf{x}_{j}+\mathbf{y} & j \in \mathcal{S} \\ \mathbf{x}_{j} & \text { otherwise }\end{cases}
$$

In other words, the function $\psi(\mathbf{X}, \mathbf{y}, \mathcal{S})$, adds $\mathbf{y}$ to the columns of $\mathbf{X}$, whose index is in set $\mathcal{S}$. In addition, $\mathbf{X}^{i^{-}}$is the matrix $\mathbf{X}$ whose $i^{t h}$ column and row is removed. We use a similar notation for a vector whose $i^{\text {th }}$ element is removed.

\section{SyStem Model AND PROBLEM Formulation}

We consider a communication system of $n$ transmitter-receiver pairs, where each transmitter aims to send data to its corresponding receiver. Each pair is named a link (user). The channels between the transmitter and receiver terminals are represented by the gain matrix $\mathbf{G}=\left[g_{i j}\right]_{n \times n}$ where $g_{i j}$ is the attenuation of the power from transmitter $j$ to receiver $i$. This attenuation can be the result of fading, shadowing, or the processing gain of the CDMA system. A white Gaussian noise with zero mean and variance $\sigma_{i}^{2}$ is added to each signal at the receiver $i$ terminal. The receivers are conventional receivers in the sense that each receiver only decodes its corresponding data and multiuser detection is not employed. Assuming the transmitters utilize Gaussian codebooks, the distribution of the interference will be Gaussian, as well. Hence, the SINR of user $i$ denoted by $\gamma_{i}$ is obtained as

$$
\gamma_{i}=\frac{g_{i i} p_{i}}{\sigma_{i}^{2}+\sum_{\substack{j=1 \\ j \neq i}}^{n} g_{i j} p_{j}}, \quad \forall i \in\{1, \ldots, n\},
$$

where $p_{i}$ is the power of transmitter $i$. In practice, the power vector $\mathbf{p}=\left[p_{i}\right]_{n \times 1}$ is subject to the trivial constraint

$$
\mathrm{p} \geq \mathbf{0}
$$

and a set of constraints in the form of

$$
\sum_{i \in \Omega_{m}} p_{i} \leq \bar{p}_{\Omega_{m}}, \quad m=1, \cdots, M
$$

where $\Omega_{m} \subseteq\{1, \ldots, n\}$ and $M$ is the number of constraints. Note that the scenarios of individual power constraints and total power constraints can be considered as special cases of (3). A power vector is said to be admissible if it satisfies the power constraints (2) and (3). 


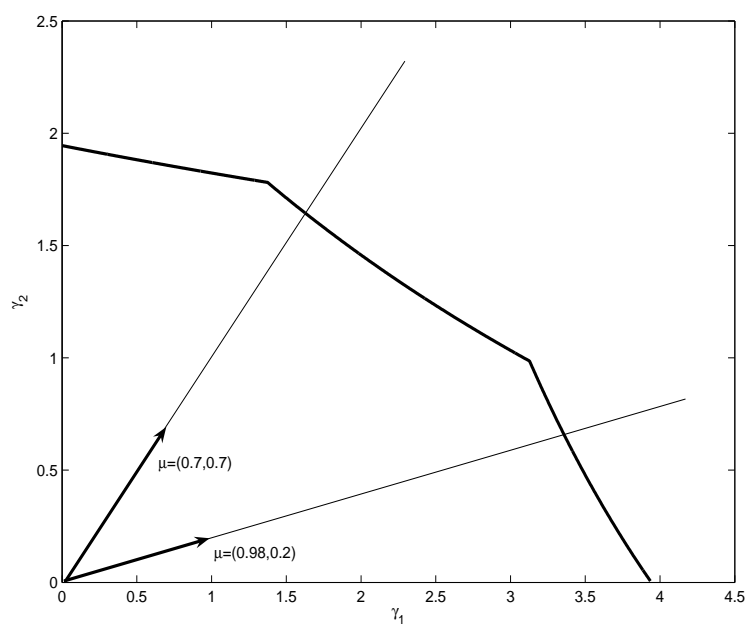

Fig. 1. The boundary of SINR Region for two-interfering links

Proposition 1 For any two power vectors $\mathbf{p}$ and $\hat{\mathbf{p}}$ that satisfy $\mathbf{0} \leq \mathbf{p} \leq \hat{\mathbf{p}}$, if $\hat{\mathbf{p}}$ is admissible then, $\mathbf{p}$ is admissible as well.

Proof: The proof follows from the fact that in the power constraints (3), all powers $p_{i}$ appear with non-negative coefficients.

The main goal is to find the feasible SINR region, i.e. the set of all points $\left(\gamma_{1}, \cdots, \gamma_{n}\right)$ which are obtained from all admissible power vectors $\mathbf{p}$. To this end, we try to find the boundary points of the feasible region. Any boundary point of the feasible region is the farthest feasible point from the origin in a direction $\boldsymbol{\mu} \geq \mathbf{0}$. If we can obtain such a point for an arbitrary $\boldsymbol{\mu}$, then the whole boundary can be obtained by changing $\boldsymbol{\mu}$ to different directions (see Fig. 1). As a result, the boundary of the SINR region is parametrically described in terms of the parameter $\boldsymbol{\mu}$.

By introducing a new variable $\gamma$ and for a unit-length vector $\boldsymbol{\mu}$, the above discussion can be formulated as the problem of finding the largest $\gamma$ such that $\gamma \boldsymbol{\mu}$ is feasible. This problem can be written as

$$
\begin{aligned}
& \max _{\mathbf{p}, \gamma} \gamma \\
& \text { s.t. } \quad \gamma_{i}=\mu_{i} \gamma, \quad i=1, \cdots, n \\
& \mathbf{p} \geq \mathbf{0} \\
& \sum_{i \in \Omega_{m}} p_{i} \leq \bar{p}_{\Omega_{m}}, \quad m=1, \cdots, M
\end{aligned}
$$


The numerical solution of this problem can be obtained through geometric programming [22], [23]; however, we propose a different approach which leads to a closed-form solution.

\section{SINR REGION CHARACTERIZATION}

The purpose of this section is to obtain a closed form expression for the boundary points of the feasible SINR region by using problem (4) and the discussion preceding it. First, we consider a special case, where there is only one power constraint involved in the problem, i.e.,

$$
\begin{array}{ll}
\max _{\mathbf{p}, \gamma} & \gamma \\
\text { s.t. } & \gamma_{i}=\mu_{i} \gamma, \quad i=1, \cdots, n \\
& \mathbf{p} \geq \mathbf{0} \\
& \sum_{i \in \Omega} p_{i} \leq \bar{p}_{\Omega},
\end{array}
$$

where $\Omega \subseteq\{1, \ldots, n\}$. Later, we show how to obtain the solution of (4) by utilizing the solution of (5).

By defining the normalized gain matrix $\mathbf{A}$ as

$$
\mathbf{A}=\left[a_{i j}\right]_{n \times n}, \quad a_{i j}= \begin{cases}\frac{g_{i j}}{g_{i i}} & i \neq j \\ 0 & i=j\end{cases}
$$

the constraint $\gamma_{i}=\mu_{i} \gamma$ in (5) is rewritten as

$$
\frac{p_{i}}{\eta_{i}+\sum_{j=1}^{n} \mu_{i} a_{i j} p_{j}}=\gamma, \quad \forall i \in\{1, \ldots, n\}
$$

where

$$
\eta_{i}=\frac{\mu_{i} \sigma_{i}^{2}}{g_{i i}}, \quad \boldsymbol{\eta}=\left[\eta_{i}\right]_{n \times 1} .
$$

After reformulating (7) in a matrix form, we have

$$
\left(\frac{1}{\gamma} \mathbf{I}-\operatorname{diag}(\boldsymbol{\mu}) \mathbf{A}\right) \mathbf{p}=\boldsymbol{\eta}
$$

This is a system of linear equations in $\mathbf{p}$. The objective is to find the maximum $\gamma$ such that the solution for this system of linear equations satisfies the power constraints of (5).

When there is no constraint on the power vector (other than the trivial constraint of $\mathbf{p} \geq \mathbf{0}$ ), it is a well-known result [12] that an SINR $\gamma$ is achievable if and only if

$$
0 \leq \gamma<\frac{1}{\lambda^{*}(\operatorname{diag}(\boldsymbol{\mu}) \mathbf{A})}
$$


where $\lambda^{*}(\cdot)$ is the Perron-Frobenius eigenvalue ${ }^{1}$ of the associated matrix [21]. We aim to obtain the range of achievable SINRs under the additional constraint $\sum_{i \in \Omega} p_{i} \leq \bar{p}_{\Omega}$.

Let us define $\mathbf{F}$ as

$$
\mathbf{F}=\mathbf{I}-\gamma \operatorname{diag}(\boldsymbol{\mu}) \mathbf{A}
$$

Then, the system of linear equations in (9) is reformulated as

$$
\mathbf{F p}=\gamma \boldsymbol{\eta},
$$

where $\boldsymbol{\eta}$ is defined in (8). According to the Cramer's rule, the solution to (12) is obtained by

$$
p_{i}=\frac{\operatorname{det}\left(\mathbf{H}^{(i)}\right)}{\operatorname{det}(\mathbf{F})}
$$

where $\mathbf{H}^{(i)}=\psi\left(\mathbf{F}, \gamma \boldsymbol{\eta}-\mathbf{f}_{i},\{i\}\right)$. Note that the numerator and the denominator in (13) are polynomials in $\gamma$. Therefore, the constraint $\sum_{i \in \Omega} p_{i} \leq \bar{p}_{\Omega}$ can be written as

$$
\frac{\sum_{i \in \Omega} \operatorname{det}\left(\mathbf{H}^{(i)}\right)}{\operatorname{det}(\mathbf{F})} \leq \bar{p}_{\Omega} \text {. }
$$

Defining $\mathrm{u}_{\Omega}(\gamma)=\bar{p}_{\Omega} \operatorname{det}(\mathbf{F})-\sum_{i \in \Omega} \operatorname{det}\left(\mathbf{H}^{(i)}\right)$ and $\mathrm{f}(\gamma)=\operatorname{det}(\mathbf{F})$, the inequality (14) is equivalent to

$$
\frac{\mathrm{u}_{\Omega}(\gamma)}{\mathrm{f}(\gamma)} \geq 0
$$

Let $\nu\left(\mathrm{u}_{\Omega}\right)$ and $\nu(\mathrm{f})$ denote the smallest positive real simple root of $\mathrm{u}_{\Omega}(\gamma)$ and $\mathrm{f}(\gamma)$, respectively. To proceed with the problem of finding the feasible range of $\gamma$, we should first find these values. The following lemma states one of the properties of matrices that will help us through the problem analysis.

Lemma 2 If square matrices $\mathbf{X}$ and $\mathbf{Y}$ differ only in column $i$, i.e., $\left\{\begin{array}{ll}\mathbf{x}_{j} \neq \mathbf{y}_{j} & j=i \\ \mathbf{x}_{j}=\mathbf{y}_{j} & j \neq i\end{array}\right.$, then

$$
\begin{aligned}
\operatorname{det}(\mathbf{X})+\operatorname{det}(\mathbf{Y}) & =\operatorname{det}\left(\psi\left(\mathbf{X}, \mathbf{y}_{i},\{i\}\right)\right) \\
& =\operatorname{det}\left(\psi\left(\mathbf{Y}, \mathbf{x}_{i},\{i\}\right)\right)
\end{aligned}
$$

Lemma 3 The polynomial $\mathrm{u}_{\Omega}(\gamma)$ can be described as

$$
\mathrm{u}_{\Omega}(\gamma)=\bar{p}_{\Omega} \operatorname{det}\left(\psi\left(\mathbf{F},-\frac{\gamma \boldsymbol{\eta}}{\bar{p}_{\Omega}}, \Omega\right)\right) \text {. }
$$

${ }^{1}$ See Theorem 4 for the definition of the Perron-Frobenius eigenvalue. 
Proof: Without loss of generality, we assume that $\Omega=\{1, \ldots, k\}$, i.e., the first $k$ users are subject to the total power constraint. From the definition of $\mathrm{u}_{\Omega}(\gamma)$ and $\mathbf{H}^{(i)}$, we have

$$
\mathrm{u}_{\Omega}(\gamma)=\bar{p}_{\Omega}\left(\operatorname{det}(\mathbf{F})-\sum_{i=1}^{k} \operatorname{det}\left(\hat{\mathbf{H}}^{(i)}\right)\right)
$$

where $\hat{\mathbf{H}}^{(i)}=\psi\left(\mathbf{F}, \frac{\gamma \boldsymbol{\eta}}{\bar{p}_{\Omega}}-\mathbf{f}_{i},\{i\}\right)$. Equation (16) is rewritten as

$$
\mathrm{u}_{\Omega}(\gamma)=\bar{p}_{\Omega}\left(\operatorname{det}(\mathbf{F})-\operatorname{det}\left(\hat{\mathbf{H}}^{(1)}\right)-\sum_{i=2}^{k} \operatorname{det}\left(\hat{\mathbf{H}}^{(i)}\right)\right) \text {. }
$$

Since $\mathbf{F}$ and $\hat{\mathbf{H}}^{(1)}$ are the same except for the first column, using Lemma 2 , we have

$$
\operatorname{det}(\mathbf{F})-\operatorname{det}\left(\hat{\mathbf{H}}^{(1)}\right)=\operatorname{det}\left(\psi\left(\mathbf{F},-\frac{\gamma \boldsymbol{\eta}}{\bar{p}_{\Omega}},\{1\}\right)\right) .
$$

On the other hand, using the fact that adding a multiple of a column to another does not change the value of the determinant, we have

$$
\operatorname{det}\left(\hat{\mathbf{H}}^{(i)}\right)=\operatorname{det}\left(\psi\left(\hat{\mathbf{H}}^{(i)},-\hat{\mathbf{h}}_{i}^{(i)},\{1, \ldots, i-1\}\right)\right) \text {. }
$$

Then, using (18) and (19) and regarding $\hat{\mathbf{h}}_{i}^{(i)}=\frac{\gamma \boldsymbol{\eta}}{\bar{p}_{\Omega}}$, we can rewrite (17) as

$$
\begin{aligned}
\mathrm{u}_{\Omega}(\gamma) & =\bar{p}_{\Omega}\left(\operatorname{det}\left(\psi\left(\mathbf{F},-\frac{\gamma \boldsymbol{\eta}}{\bar{p}_{\Omega}},\{1\}\right)\right)\right. \\
& \left.-\sum_{i=2}^{k} \operatorname{det}\left(\psi\left(\hat{\mathbf{H}}^{(i)},-\frac{\gamma \boldsymbol{\eta}}{\bar{p}_{\Omega}},\{1, \ldots, i-1\}\right)\right)\right) .
\end{aligned}
$$

Since $\mathbf{F}$ and $\hat{\mathbf{H}}^{(i)}$ are the same except for the column $i$, we can easily see that the matrices $\psi\left(\mathbf{F},-\frac{\gamma \boldsymbol{\eta}}{\bar{p}_{\Omega}},\{1, \ldots, i-1\}\right)$ and $\psi\left(\hat{\mathbf{H}}^{(i)},-\frac{\gamma \boldsymbol{\eta}}{\bar{p}_{\Omega}},\{1, \ldots, i-1\}\right)$ are the same except for the $i^{t h}$ column. Therefore,

$$
\begin{aligned}
& \operatorname{det}\left(\psi\left(\mathbf{F},-\frac{\gamma \boldsymbol{\eta}}{\bar{p}_{\Omega}},\{1, \ldots, i-1\}\right)\right) \\
& -\operatorname{det}\left(\psi\left(\hat{\mathbf{H}}^{(i)},-\frac{\gamma \boldsymbol{\eta}}{\bar{p}_{\Omega}},\{1, \ldots, i-1\}\right)\right) \\
& =\operatorname{det}\left(\psi\left(\mathbf{F},-\frac{\gamma \boldsymbol{\eta}}{\bar{p}_{\Omega}},\{1, \ldots, i\}\right)\right) .
\end{aligned}
$$

By successively applying this procedure to (20), the claim is proved.

The representation of $\mathrm{u}_{\Omega}(\gamma)$ in Lemma 3 lends itself to finding the smallest positive simple root of $\mathrm{u}_{\Omega}(\gamma)$. To this end, we need the Perron-Frobenius theorem for irreducible matrices. A square 
non-negative matrix $\mathbf{X}$ is said to be irreducible if for every pair $i, j$ of its index set, there exists a positive integer $m \equiv m(i, j)$ such that $x_{i j}^{(m)}>0$, where $x_{i j}^{(m)}$ is the $(i, j)^{t h}$ element of $\mathbf{X}^{m}$ [21].

Theorem 4 [21] (The Perron-Frobenius Theorem for irreducible matrices) Suppose $\mathbf{X}$ is an $m \times m$ irreducible non-negative matrix. Then there exists an eigenvalue $\lambda^{*}(\mathbf{X})$ (Perron-Frobenius eigenvalue or PF-eigenvalue) such that

(i) $\lambda^{*}(\mathbf{X})>0$ and it is real.

(ii) $\lambda^{*}(\mathbf{X}) \geq|\lambda(\mathbf{X})|$ for any eigenvalue $\lambda(\mathbf{X}) \neq \lambda^{*}(\mathbf{X})$.

(iii) If $\mathbf{X} \geq \mathbf{Y} \geq \mathbf{0}$, then $\lambda^{*}(\mathbf{X}) \geq|\lambda(\mathbf{Y})|$ for any eigenvalue of $\mathbf{Y}$.

(iv) $\lambda^{*}(\mathbf{X})$ is a simple root of the characteristic polynomial of $\mathbf{X}$.

Lemma 5 The smallest positive root of $\mathrm{u}_{\Omega}(\gamma)$ and $\mathrm{f}(\gamma)$, respectively, are

$$
\begin{aligned}
\nu\left(\mathrm{u}_{\Omega}\right) & =\frac{1}{\lambda^{*}\left(\psi\left(\operatorname{diag}(\boldsymbol{\mu}) \mathbf{A}, \frac{\boldsymbol{\eta}}{\bar{p}_{\Omega}}, \Omega\right)\right)}, \\
\nu(\mathrm{f}) & =\frac{1}{\lambda^{*}(\operatorname{diag}(\boldsymbol{\mu}) \mathbf{A})} .
\end{aligned}
$$

Proof: From Lemma 3, we have

$$
\begin{aligned}
\mathrm{u}_{\Omega}(\gamma) & =\bar{p}_{\Omega} \operatorname{det}\left(\psi\left(\mathbf{F},-\frac{\gamma \boldsymbol{\eta}}{\bar{p}_{\Omega}}, \Omega\right)\right) \\
& =\bar{p}_{\Omega} \operatorname{det}\left(\psi\left(\mathbf{I}-\gamma \operatorname{diag}(\boldsymbol{\mu}) \mathbf{A},-\frac{\gamma \boldsymbol{\eta}}{\bar{p}_{\Omega}}, \Omega\right)\right) \\
& =\bar{p}_{\Omega} \gamma^{n} \operatorname{det}\left(\psi\left(\frac{1}{\gamma} \mathbf{I}-\operatorname{diag}(\boldsymbol{\mu}) \mathbf{A},-\frac{\boldsymbol{\eta}}{\bar{p}_{\Omega}}, \Omega\right)\right) \\
& =\bar{p}_{\Omega} \gamma^{n} \operatorname{det}\left(\frac{1}{\gamma} \mathbf{I}-\psi\left(\operatorname{diag}(\boldsymbol{\mu}) \mathbf{A}, \frac{\boldsymbol{\eta}}{\bar{p}_{\Omega}}, \Omega\right)\right) .
\end{aligned}
$$

Consequently, $\frac{\mathrm{u}_{\Omega}(\gamma)}{\bar{p}_{\Omega}}$ is the reciprocal of the characteristic polynomial of $\psi\left(\operatorname{diag}(\boldsymbol{\mu}) \mathbf{A}, \frac{\boldsymbol{\eta}}{\bar{p}_{\Omega}}, \Omega\right)$. Therefore, the roots of $\mathrm{u}_{\Omega}(\gamma)$ are equal to the inverse of the eigenvalues of $\psi\left(\operatorname{diag}(\boldsymbol{\mu}) \mathbf{A}, \frac{\boldsymbol{\eta}}{\bar{p}_{\Omega}}, \Omega\right)$. Assuming all channel gains $g_{j i}$ and all weighting coefficients $\mu_{i}$ are positive, it can be verified that all elements of $\psi^{2}\left(\operatorname{diag}(\boldsymbol{\mu}) \mathbf{A}, \frac{\boldsymbol{\eta}}{\bar{p}_{\Omega}}, \Omega\right)$ are positive. This means $\psi\left(\operatorname{diag}(\boldsymbol{\mu}) \mathbf{A}, \frac{\boldsymbol{\eta}}{\bar{p}_{\Omega}}, \Omega\right)$ is an irreducible matrix. Hence, according to Theorem 4, the PF-eigenvalue of this matrix is real and positive and has the largest norm among all eigenvalues. Also, it is a simple root of 
the characteristic polynomial of the aforementioned matrix. Therefore, the inverse of the PFeigenvalue of $\psi\left(\operatorname{diag}(\boldsymbol{\mu}) \mathbf{A}, \frac{\boldsymbol{\eta}}{\bar{p}_{\Omega}}, \Omega\right)$ gives the smallest positive simple root of $\mathrm{u}_{\Omega}(\gamma)$ and the first part of the claim is proved.

To find $\nu(f)$, note that

$$
\begin{aligned}
\mathrm{f}(\gamma) & =\operatorname{det}(\mathbf{F})=\operatorname{det}(\mathbf{I}-\gamma \operatorname{diag}(\boldsymbol{\mu}) \mathbf{A}) \\
& =\gamma^{n} \operatorname{det}\left(\frac{1}{\gamma} \mathbf{I}-\operatorname{diag}(\boldsymbol{\mu}) \mathbf{A}\right),
\end{aligned}
$$

where the definition of $\mathbf{F}$ in (11) has been used. Therefore, $f(\gamma)$ is the reciprocal of the characteristic polynomial of $\operatorname{diag}(\boldsymbol{\mu})$ A. Hence, with the same argument as for $\mathrm{u}_{\Omega}(\gamma)$, it can be shown that the inverse of the PF-eigenvalue of $\operatorname{diag}(\boldsymbol{\mu}) \mathbf{A}$ gives the smallest positive simple root of $f(\gamma)$. This completes the proof.

Lemma 6 The smallest positive root of $\mathrm{u}_{\Omega}(\gamma)$ and $\mathrm{f}(\gamma)$ satisfy $\nu\left(\mathrm{u}_{\Omega}\right) \leq \nu(\mathrm{f})$.

Proof: Since $\psi\left(\operatorname{diag}(\boldsymbol{\mu}) \mathbf{A}, \frac{\boldsymbol{\eta}}{\bar{p}_{\Omega}}, \Omega\right) \geq \operatorname{diag}(\boldsymbol{\mu}) \mathbf{A} \geq \mathbf{0}$ and both are irreducible, using Theorem 4, we have

$$
\lambda^{*}\left(\psi\left(\operatorname{diag}(\boldsymbol{\mu}) \mathbf{A}, \frac{\boldsymbol{\eta}}{\bar{p}_{\Omega}}, \Omega\right)\right) \geq \lambda^{*}(\operatorname{diag}(\boldsymbol{\mu}) \mathbf{A}) .
$$

According to Lemma 5, this inequality can be rewritten as

$$
\frac{1}{\nu\left(\mathrm{u}_{\Omega}\right)} \geq \frac{1}{\nu(\mathrm{f})}
$$

This completes the proof.

Theorem 7 In a system of $n$ interfering links and the normalized gain matrix $\mathrm{A}$, under the power constraints,

$$
\mathbf{p} \geq \mathbf{0}, \quad \sum_{i \in \Omega} p_{i} \leq \bar{p}_{\Omega}, \quad \Omega \subseteq\{1, \ldots, n\}
$$

an SINR $\gamma$ is feasible if and only if

$$
0 \leq \gamma \leq \frac{1}{\lambda^{*}\left(\psi\left(\operatorname{diag}(\boldsymbol{\mu}) \mathbf{A}, \frac{\boldsymbol{\eta}}{\bar{p}_{\Omega}}, \Omega\right)\right)}
$$

To prove the theorem, we need the following lemma. 
Lemma 8 Consider two SINR vectors $\gamma$ and $\hat{\gamma}$, which correspond to the power vectors $\mathbf{p}$ and $\hat{\mathbf{p}}$, respectively. A necessary condition for the inequality $\gamma \leq \hat{\gamma}$ to hold is that $\mathbf{p} \leq \hat{\mathbf{p}}$.

Proof: See the Appendix.

Proof of Theorem 7: According to Lemma 5, (24) is equivalent to

$$
0 \leq \gamma \leq \nu\left(\mathrm{u}_{\Omega}\right)
$$

Also, recall that the total power constraint is equivalent to (15).

For the achievability part, we should show that any $\gamma$ satisfying (25) corresponds to an admissible power vector. Recall that the condition for the positivity of the power vector is given in (10) and the total power constraint is equivalent to (15). The positivity constraint is guaranteed due to the fact that the right-hand-side of (10) equals $\nu(\mathrm{f})$ and $\nu\left(\mathrm{u}_{\Omega}\right) \leq \nu(\mathrm{f})$ (see Lemma 6). To prove that inequality (15) holds, note that $\mathrm{u}_{\Omega}(0)>0$ and $\mathrm{f}(0)>0$. Since $\nu\left(\mathrm{u}_{\Omega}\right) \leq \nu(\mathrm{f})$ (see Lemma 6), both polynomials $\mathrm{u}_{\Omega}(\gamma)$ and $\mathrm{f}(\gamma)$ are non-negative for $\gamma \leq \nu\left(\mathrm{u}_{\Omega}\right)$, which means their ratio is non-negative as well. This completes the proof for achievability.

For the converse, we should show that any $\gamma, \gamma>\nu\left(\mathrm{u}_{\Omega}\right)$, is not feasible. From (10), the constraint $\mathbf{p} \geq \mathbf{0}$ guarantees that $\gamma \geq \nu(\mathrm{f})$ is not feasible. If $\nu\left(\mathrm{u}_{\Omega}\right)=\nu(\mathrm{f})$, the proof is complete. For the case that $\nu\left(\mathrm{u}_{\Omega}\right)<\nu(\mathrm{f})$, we should prove that the values of $\gamma$ in the range $\nu\left(\mathrm{u}_{\Omega}\right)<\gamma<$ $\nu(\mathrm{f})$ are not feasible. First, note that $\frac{\mathrm{u}_{\Omega}(\gamma)}{\mathrm{f}(\gamma)}$ is a continuous function of $\gamma$ and $\nu\left(\mathrm{u}_{\Omega}\right)$ is its smallest positive simple root. Hence, there exists an $\epsilon>0$, such that for $\nu\left(\mathrm{u}_{\Omega}\right)<\gamma<\nu\left(\mathrm{u}_{\Omega}\right)+\epsilon$, we have $\frac{\mathrm{u}_{\Omega}(\gamma)}{\mathrm{f}(\gamma)}<0$ and the constraint (15) is violated. Hence, these values of $\gamma$ are not achievable. Now, assume by contradiction that a $\hat{\gamma}$ satisfying $\nu\left(\mathrm{u}_{\Omega}\right)+\epsilon<\hat{\gamma}<\nu(\mathrm{f})$ is achievable and corresponds to the power vector $\hat{\mathbf{p}}$. From (10), any $\gamma$ that satisfies $\gamma<\hat{\gamma}$, corresponds to a power vector $\mathbf{p}>\mathbf{0}$. Due to Lemma 8 , we have $\mathbf{p} \leq \hat{\mathbf{p}}$. Since $\hat{\mathbf{p}}$ is an admissible power vector, according to Proposition 1, $\mathbf{p}$ is admissible as well. This means any $\gamma, \gamma<\hat{\gamma}$, is achievable. This is in contradiction to the fact that $\nu\left(\mathrm{u}_{\Omega}\right)<\gamma<\nu\left(\mathrm{u}_{\Omega}\right)+\epsilon$ is not achievable.

Theorem 7 describes the feasible SINR region when there is only one power constraint as shown in problem (5). The next theorem shows how to utilize the result of Theorem 7 to obtain the solution of the original problem (4), where multiple power constraints exist.

Theorem 9 Assume $\gamma^{*}$ is the solution of (4) and $\gamma_{m}^{*}$ is the solution of the same problem when 
only the mth power constraint of (4) exists. Then, we have

$$
\gamma^{*}=\min _{m} \gamma_{m}^{*}
$$

Proof: For a general maximization problem with $M$ constraints, it is obvious that the solution is at most equal to the minimum of the solutions of the same problems with single constraints, i.e.

$$
\gamma^{*} \leq \min _{m} \gamma_{m}^{*}
$$

By defining $m^{*}=\arg \min _{m} \gamma_{m}$, (27) can be rewritten as

$$
\gamma^{*} \leq \gamma_{m^{*}}^{*}
$$

Assume $\mathbf{p}_{m}^{*}$ is the power vector corresponding to $\gamma_{m}^{*}$ for all $m \in\{1, \cdots, M\}$. According to Lemma 8, we have $\mathbf{p}_{m^{*}}^{*} \leq \mathbf{p}_{m}^{*}$ for all $m \in\{1, \cdots, M\}$. Hence, $\mathbf{p}_{m^{*}}^{*}$ is an admissible power vector for problem (4). As a result, we have

$$
\gamma^{*} \geq \gamma_{m^{*}}^{*}
$$

The theorem is proved by comparing (28) and (29).

In the common scenario when the power of individual users and the total power are constrained, Theorems 7 and 9 yield the following result on the maximum achievable SINR .

Corollary 10 The maximum achievable $\gamma$ in (4), where power vector is subject to the following constraints,

$$
\mathbf{p} \geq \mathbf{0}, \quad \mathbf{p} \leq \overline{\mathbf{p}}, \quad \sum_{i=1}^{n} p_{i} \leq \bar{p}_{t}
$$

is equal to

$$
\begin{aligned}
\gamma^{*}=\min & \left\{\frac{1}{\lambda^{*}\left(\psi\left(\operatorname{diag}(\boldsymbol{\mu}) \mathbf{A}, \frac{\boldsymbol{\eta}}{\bar{p}_{t}},\{1, \ldots, n\}\right)\right)},\right. \\
& \frac{1}{\lambda^{*}\left(\psi\left(\operatorname{diag}(\boldsymbol{\mu}) \mathbf{A}, \frac{\boldsymbol{\eta}}{\bar{p}_{1}},\{1\}\right)\right)}, \frac{1}{\lambda^{*}\left(\psi\left(\operatorname{diag}(\boldsymbol{\mu}) \mathbf{A}, \frac{\boldsymbol{\eta}}{\bar{p}_{2}},\{2\}\right)\right)}, \\
& \left.\ldots, \frac{1}{\lambda^{*}\left(\psi\left(\operatorname{diag}(\boldsymbol{\mu}) \mathbf{A}, \frac{\boldsymbol{\eta}}{\bar{p}_{n}},\{n\}\right)\right)}\right\}
\end{aligned}
$$




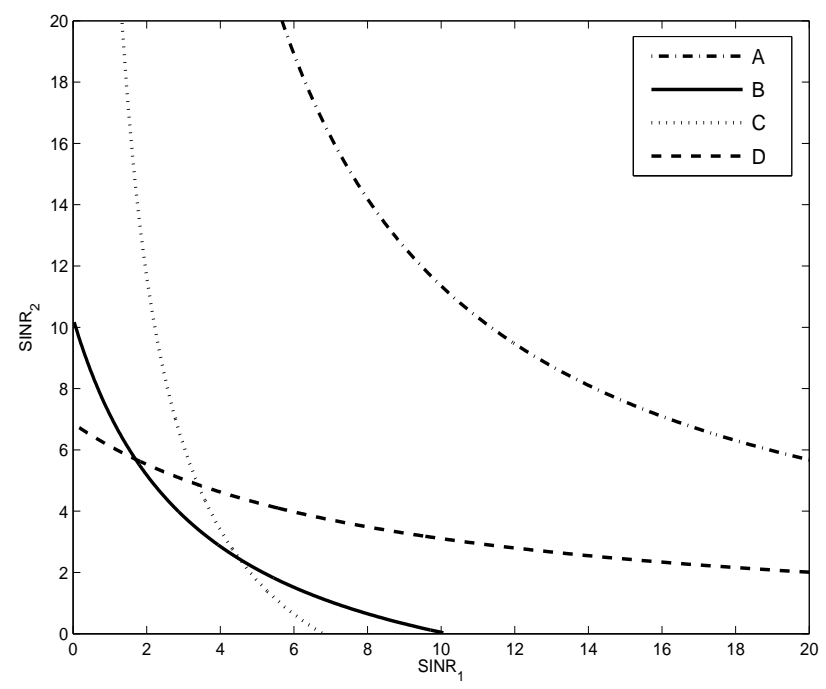

Fig. 2. The SINR region for 2 interfering links with the following constraints on the power, A: $p_{1} \geq 0, p_{2} \geq 0$, B: $p_{1}+p_{2} \leq \bar{p}_{t}, p_{1} \geq 0, p_{2} \geq 0$ C: $0 \leq p_{1} \leq \bar{p}_{1}, p_{2} \geq 0$, D: $0 \leq p_{2} \leq \bar{p}_{2}, p_{1} \geq 0$

The boundary of the SINR region in any direction can be obtained by choosing $\boldsymbol{\mu}$, accordingly. Due to the explicit relationship between the SINR and the rate in Gaussian channels, obtaining the SINR region in these channels amounts to the rate region characterization. As an example, Fig. 2 and Fig. 3, respectively, depict the SINR region and the rate region of a system with the gain matrix $\mathrm{G}$ as

$$
\mathbf{G}=\left[\begin{array}{ll}
0.6791 & 0.0999 \\
0.0411 & 0.6864
\end{array}\right]
$$

while the power of individual users and the total power are upper-bounded by $\bar{p}_{1}=0.8, \quad \bar{p}_{2}=$ $1, \quad \bar{p}_{t}=1.4$, and $\sigma_{1}^{2}=\sigma_{2}^{2}=10^{-1}$.

\section{TIme-VARYing Channel}

So far, we have assumed that the channel gains are fixed with time. However, in practice, channel gains vary with time due to the users movement or environment changes.

In this section, we consider a communication system with $n$ interfering links whose channel gain matrix is randomly selected from a finite set $\left\{\mathbf{G}_{1}, \ldots, \mathbf{G}_{L}\right\}$ with probabilities $\rho_{1}, \ldots, \rho_{L}$, respectively. The goal is to characterize the SINR region $\mathcal{F}$ which is achievable regardless of the channel state. 


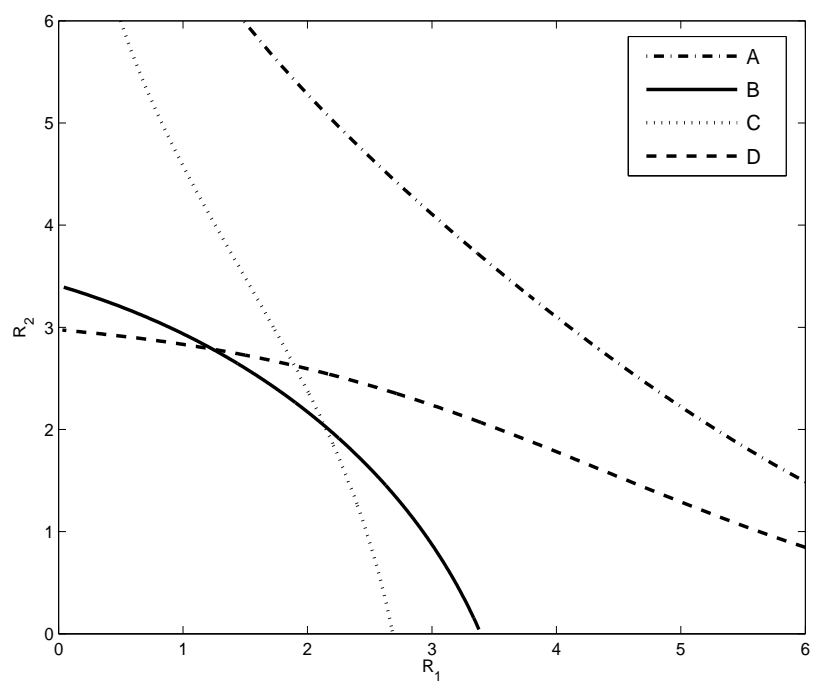

Fig. 3. The rate region for a 2 -interfering-link system with the following constraints on the power, A: $p_{1} \geq 0$, $p_{2} \geq 0, \mathrm{~B}: p_{1}+p_{2} \leq \bar{p}_{t}, p_{1} \geq 0, p_{2} \geq 0 \mathrm{C}: 0 \leq p_{1} \leq \bar{p}_{1}, p_{2} \geq 0, \mathrm{D}: 0 \leq p_{2} \leq \bar{p}_{2}, p_{1} \geq 0$

If the transmitters are subject to instantaneous power constraints, as in the previous section, the achievable SINR region is the intersection of the achievable regions in each channel realization. In other words, assuming $\mathcal{F}_{l}$ denotes the achievable region in state $l$, we have

$$
\mathcal{F}=\bigcap_{l=1}^{L} \mathcal{F}_{l} .
$$

A more common scenario for time-varying channels is the case where the average transmit powers are constrained. In this case, the transmitters enjoy the flexibility of using less power when the channel conditions are good and save the power for unfavorable channel states. Fig. 4 provides a comparison for the two scenarios with an example of a system with two interfering links. It is observed that the feasible region in the instantaneous power constrained scenario is a subset of the feasible region in the average power constrained scenario.

In [16], it is shown that in a point-to-point system, the maximum zero-outage SINR is equal to $\frac{\bar{p}}{E_{l}\left[1 / g_{l}\right]}$, where $\bar{p}$ is the average transmit signal power and $g_{l}$ is the power gain of the channel in the $l$ th realization. In accordance with the concept of zero-outage SINR, in this section we consider the zero-outage SINR region defined as the set of SINRs which are achievable regardless of the channel realization. The objective is to characterize the zero-outage SINR region when we have $n$ interfering links with finite number of channel realizations and the average transmitter 


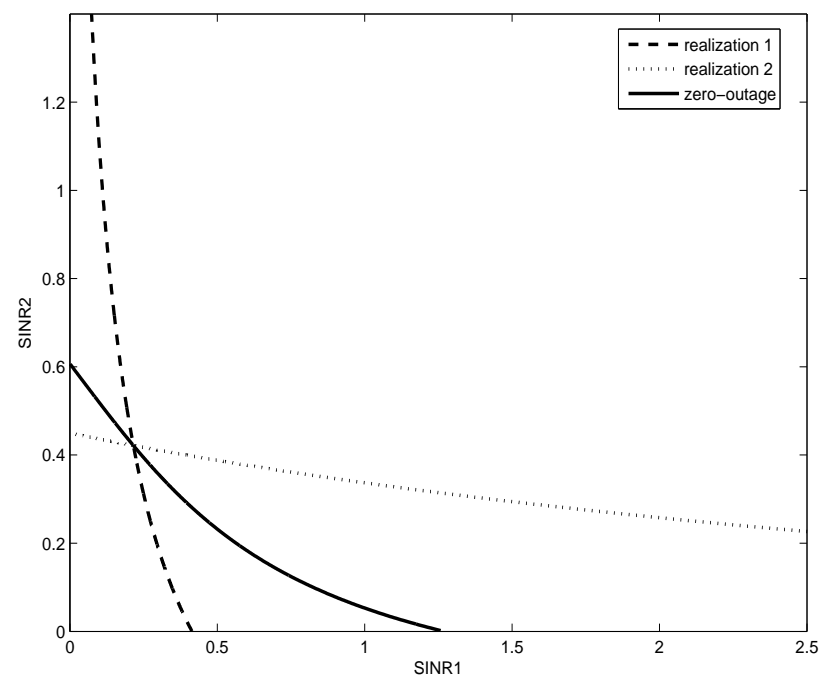

Fig. 4. The SINR region for a two-interfering-link system $\rho_{1}=0.3, \quad \rho_{2}=0.7, \quad \bar{p}_{t}=1$

powers are subject to some linear constraints. Following the same discussions as in the previous sections, it is concluded that this problem is equivalent to finding the maximum $\gamma$ which is achievable by all users in all channel states, while the average power of the users are constrained. In mathematical notations, we have the following optimization problem

$$
\begin{aligned}
& \max _{p_{i, l}, \gamma} \gamma \\
& \text { s.t. } \quad \gamma_{i, l} \geq \mu_{i} \gamma, \quad \forall i, l, \quad i \in\{1, \ldots, n\}, \quad l \in\{1, \ldots, L\} \\
& p_{i, l} \geq 0, \quad \forall i, l, \quad i \in\{1, \ldots, n\}, \quad l \in\{1, \ldots, L\} \\
& E_{l}\left[\sum_{i \in \Omega} p_{i, l}\right] \leq \bar{p}_{\Omega},
\end{aligned}
$$

where $\gamma_{i, l}$ and $p_{i, l}$ are respectively the SINR and the power of transmitter $i$, when the channel gain matrix is $\mathbf{G}_{l}$. Note that the constraint $\gamma_{i, l} \geq \mu_{i} \gamma$ guarantees that the SINR of user $i$ is greater than $\mu_{i} \gamma$, regardless of the channel state $l$. If there were more power constraints in (31), similar to the previous section, the solution would be equal to the minimum solution of the same problems with separate single power constraints. Hence, in this section we solely concentrate on the above problem with a single power constraint.

We define an expanded system including $n L$ users with block diagonal matrices $\tilde{\mathbf{G}}$ and $\tilde{\mathbf{A}}$ as the channel gain matrix and the normalized gain matrix, respectively. Let $\mathbf{A}_{l}$ denote the normalized gain matrix in the state $l \in\{1, \ldots, L\}$. Matrices $\tilde{\mathbf{G}}$ and $\tilde{\mathbf{A}}$ are block diagonal matrices, where the $l^{t h}$ submatrix on the diagonal is $\mathbf{G}_{l}$ and $\mathbf{A}_{l}$, respectively. It is clear that 
block diagonal format of these matrices reflects the fact that there is no interference between the virtual links associated with different states. In the new system, $p_{i+(l-1) n}$ denotes the power of transmitter $i$, when the channel gain matrix is $\mathbf{G}_{l}$. Similar to the previous discussions, the requirements on these links form a system of linear equations with the following formulation in a matrix form,

$$
\left(\frac{1}{\gamma} \mathbf{I}_{n L \times n L}-\operatorname{diag}\left(\mathbf{1}_{L \times 1} \otimes \boldsymbol{\mu}\right) \tilde{\mathbf{A}}\right) \mathbf{p}=\boldsymbol{\eta},
$$

where

$$
\eta_{i+(l-1) n}=\frac{\mu_{i} \sigma_{i}^{2}}{g_{i+(l-1) n, i+(l-1) n}}, i \in\{1, \ldots, n\}, l \in\{1, \ldots, L\} .
$$

Similar to (11), we define $\mathbf{F}$ as

$$
\mathbf{F}=\frac{1}{\gamma} \mathbf{I}_{n L \times n L}-\operatorname{diag}\left(\mathbf{1}_{L \times 1} \otimes \boldsymbol{\mu}\right) \tilde{\mathbf{A}} .
$$

Then, we have

$$
\mathbf{F p}=\gamma \boldsymbol{\eta}
$$

Using Cramer's rule, we have

$$
p_{i+(l-1) n}=\frac{\operatorname{det}\left(\mathbf{H}^{(i+(l-1) n)}\right)}{\operatorname{det}(\mathbf{F})},
$$

where $\mathbf{H}^{(i+(l-1) n)}=\psi\left(\mathbf{F}, \gamma \boldsymbol{\eta}-\mathbf{f}_{i+(l-1) n},\{i+(l-1) n\}\right)$. The average of the total power of the users in $\Omega$ is equivalent to

$$
\begin{aligned}
E_{l}\left[\sum_{i \in \Omega} p_{i+(l-1) n}\right] & =\sum_{l=1}^{L} \rho_{l} \sum_{i \in \Omega} p_{i+(l-1) n} \\
& =\sum_{l=1}^{L} \rho_{l} \sum_{i \in \Omega} \frac{\operatorname{det}\left(\mathbf{H}^{(i+(l-1) n)}\right)}{\operatorname{det}(\mathbf{F})} \\
& =\frac{1}{\operatorname{det}(\mathbf{F})} \sum_{l=1}^{L} \rho_{l} \sum_{i \in \Omega} \operatorname{det}\left(\mathbf{H}^{(i+(l-1) n)}\right) .
\end{aligned}
$$

Based on (33), we define

$$
\mathrm{u}_{\Omega}(\gamma)=\bar{p}_{\Omega} \operatorname{det}(\mathbf{F})-\sum_{l=1}^{L} \rho_{l} \sum_{i \in \Omega} \operatorname{det}\left(\mathbf{H}^{(i+(l-1) n)}\right)
$$

and

$$
\mathrm{f}(\gamma)=\operatorname{det}(\mathbf{F})
$$


Therefore, the constraint in $E_{l}\left[\sum_{i \in \Omega} p_{i, l}\right] \leq \bar{p}_{\Omega}$ is equivalent to

$$
\frac{\mathrm{u}_{\Omega}(\gamma)}{\mathrm{f}(\gamma)} \geq 0
$$

Like before, it is easy to show that the maximum achievable SINR satisfying the power constraints of $(31)$ is

$$
\gamma^{*}=\min \left\{\nu(\mathrm{f}), \nu\left(\mathrm{u}_{\Omega}\right)\right\}
$$

To simplify $\mathrm{u}_{\Omega}(\gamma)$, we have

$$
\begin{aligned}
\mathrm{u}_{\Omega}(\gamma) & =\bar{p}_{\Omega} \operatorname{det}(\mathbf{F})-\sum_{l=1}^{L} \rho_{l} \sum_{i \in \Omega} \operatorname{det}\left(\mathbf{H}^{(i+(l-1) n)}\right) \\
& =\bar{p}_{\Omega}\left(\operatorname{det}(\mathbf{F})-\sum_{l=1}^{L} \sum_{i \in \Omega} \operatorname{det}\left(\hat{\mathbf{H}}^{(i+(l-1) n)}\right)\right)
\end{aligned}
$$

where $\hat{\mathbf{H}}^{(i+(l-1) n)}$ is $\mathbf{H}^{(i+(l-1) n)}$ whose column $i+(l-1) n$ is multiplied by $\frac{\rho_{l}}{\bar{p}_{\Omega}}$. Using the same procedure as before, we obtain

$$
\mathrm{u}_{\Omega}(\gamma)=\bar{p}_{\Omega} \operatorname{det}(\mathbf{F}-\mathbf{D})
$$

where

$$
\mathbf{D}=\sum_{l=1}^{L} \psi\left(\mathbf{0}_{n L \times n L}, \frac{\rho_{l} \gamma \boldsymbol{\eta}}{\bar{p}_{\Omega}},\{i+(l-1) n: i \in \Omega\}\right)
$$

According to Theorem 4, it is easy to see that

$$
\nu\left(\mathrm{u}_{\Omega}\right)=\frac{1}{\lambda^{*}\left(\operatorname{diag}\left(\mathbf{1}_{L \times 1} \otimes \boldsymbol{\mu}\right) \tilde{\mathbf{A}}+\sum_{l=1}^{L} \psi\left(\mathbf{0}_{n L \times n L}, \frac{\rho_{l} \boldsymbol{\eta}}{\bar{p}_{\Omega}},\{i+(l-1) n: i \in \Omega\}\right)\right)} .
$$

and

$$
\nu(\mathrm{f})=\frac{1}{\lambda^{*}\left(\operatorname{diag}\left(\mathbf{1}_{L \times 1} \otimes \boldsymbol{\mu}\right) \tilde{\mathbf{A}}\right)} .
$$

Therefore, using Theorem 4 and equation (34), we have the following theorem.

Theorem 11 The maximum achievable $\gamma$ in a time-varying communication system with $n$ interfering links and probability vector $\boldsymbol{\rho}_{L \times 1}$, with the power constraints 


$$
\begin{aligned}
& p_{i, l} \geq 0, \quad \forall i, l, \quad i \in\{1, \ldots, n\}, \quad l \in\{1, \ldots, L\} \\
& E_{l}\left[\sum_{i \in \Omega} p_{i, l}\right] \leq \bar{p}_{\Omega}
\end{aligned}
$$

is equal to

$$
\gamma^{*}=\frac{1}{\lambda^{*}\left(\operatorname{diag}\left(\mathbf{1}_{L \times 1} \otimes \boldsymbol{\mu}\right) \tilde{\mathbf{A}}+\sum_{l=1}^{L} \psi\left(\mathbf{0}_{n L \times n L}, \frac{\rho_{l} \boldsymbol{\eta}}{\bar{p}_{\Omega}},\{i+(l-1) n: i \in \Omega\}\right)\right)},
$$

where $\tilde{\mathbf{A}}$ is an $n L \times n L$ block diagonal matrix whose $l^{\text {th }}$ diagonal submatrix is the normalized gain matrix at the state $l$.

\section{Removal Algorithm}

The solution of problem 4 for $\mu_{1}=\mu_{2}=\ldots=\mu_{n}$ corresponds to a specific point on the SINR region, where all users have the same SINR. Sometimes the required SINR is above this point and accordingly it is not achievable. Therefore, like any opportunistic scheduling problem, a subset of users should be serviced and the rest are serviced in a later time-slot when the channel gains are changed. This approach exploits the channel variations and schedules the users that are in a better channel condition for transmission to improve the performance.

To find the largest set of active users satisfying the SINR requirement, we have to examine all the combinations of the users and select a feasible subset with the maximum cardinality. Clearly, this scheme is computationally exponential. As a suboptimum alternative scheme, we propose a greedy removal algorithm. The main idea behind the presented algorithm is as follows. At each step, if the active users do not satisfy the required SINR, one user is removed. This user is the one which provides the highest increase in the maximum achievable SINR if it is removed. We call this user the worst user. The proposed algorithm is presented for different sorts of power constraints.

According to (10) and Theorem 7, in general, the maximum $\gamma$ is equal to the inverse of the PF-eigenvalue of a matrix $\mathbf{X}$, i.e., $\gamma^{*}=\frac{1}{\lambda^{*}(\mathbf{X})}$. In a system with a large number of users, computing the PF-eigenvalue is computationally extensive. In this case, it is beneficial to use 
an approximation of the PF-eigenvalue as follows. According to [24], the eigenvalues of the matrices $\mathbf{X}^{q}$ and $\mathbf{X}$ are related as

$$
\lambda\left(\mathbf{X}^{q}\right)=\lambda^{q}(\mathbf{X})
$$

Furthermore, we have

$$
\operatorname{Tr}\left(\mathbf{X}^{q}\right)=\sum_{i} \lambda_{i}^{q}
$$

where $\lambda_{i}=\lambda_{i}(\mathbf{X})$ is the $i$ th eigenvalue of the matrix $\mathbf{X}$. Since the PF-eigenvalue of an irreducible matrix has the largest norm among all the eigenvalues of that matrix [24], we can approximate $\lambda^{* q}(\mathbf{X})$ with the $\operatorname{Tr}\left(\mathbf{X}^{q}\right)$, i.e., $\lambda^{* q}(\mathbf{X}) \approx \operatorname{Tr}\left(\mathbf{X}^{q}\right)$. This approximation is stronger if the power $q$ is larger. However, the simulation results show that $q=2$ yields a very good approximation of the exact value in our problem. Therefore, we use

$$
\gamma^{*} \approx \frac{1}{\sqrt{\operatorname{Tr}\left(\mathbf{X}^{2}\right)}}
$$

as an approximate value for $\gamma^{*}$. In what follows, we investigate the problem of user removal for two more common cases of power constraints and present an efficient algorithm for each case.

\section{Case One: Constraints on the Power of Individual Transmitters}

Assume there is a constraint on the power of individual transmitters. We design an efficient suboptimal algorithm to find the maximum cardinality subset of the users satisfying a minimum SINR requirement. We define the matrix $\psi^{i^{-}}\left(\mathbf{A}, \frac{\boldsymbol{\eta}}{\bar{p}_{j}},\{j\}\right)$ as the matrix $\psi\left(\mathbf{A}, \frac{\boldsymbol{\eta}}{\bar{p}_{j}},\{j\}\right)$ whose $i^{\text {th }}$ column and row are removed. Therefore, the worst link is

$$
\hat{i}=\arg \max _{i} \gamma^{* i^{-}}
$$

where

$$
\gamma^{* i^{-}}=\min _{\substack{j \neq i \\ j \neq i}} \frac{1}{\lambda^{*}\left(\psi^{i^{-}}\left(\mathbf{A}, \frac{\boldsymbol{\eta}}{\bar{p}_{j}},\{j\}\right)\right)}
$$

is the maximum achievable SINR when user $i$ is removed. Equation (37) is obtained from (26). The users are removed one by one based on (36) until all of the active users satisfy the rate requirement. We call this algorithm the Removal Algorithm II-A. 
To reduce the complexity of this algorithm, which is due to the calculation of the PFeigenvalue, we use the following approximation scheme. According to (35) and (37), we have

$$
\gamma^{* i^{-}} \approx \min _{\substack{j \neq i \\ j \neq i}} \frac{1}{\sqrt{\operatorname{Tr}\left(\psi^{i-2}\left(\mathbf{A}, \frac{\boldsymbol{\eta}}{\bar{p}_{j}},\{j\}\right)\right)}}
$$

By some straightforward calculations, this approximation can be rewritten as

$$
\gamma^{* i^{-}} \approx \min _{j} \frac{1}{\sqrt{w_{i j}}},
$$

where

$$
w_{i j}= \begin{cases}\left(\frac{\eta_{j}}{\bar{p}_{j}}\right)^{2}+\sum_{\substack{k=1 \\ k \neq i}}^{n} \sum_{\substack{l=1 \\ l \neq i}}^{n} a_{k l} a_{l k}+2 \sum_{\substack{k=1 \\ k \neq i}}^{n} \frac{\eta_{k}}{\bar{p}_{j}} a_{j k} & j \neq i \\ 0 & j=i\end{cases}
$$

According to [25], equation (36) can be simplified to

$$
\hat{i}=\arg \min _{i} \max _{j} w_{i j}
$$

Based on this result, the following algorithm is developed when the required SINR is $\gamma_{t h}$.

Removal Algorithm II-B

1) Set $\mathbf{A}$ as in (6), $\overline{\mathbf{p}}=\left[\bar{p}_{i}\right], m=n, \mathcal{R}=\varnothing$, and $\mathbf{v}=[1,2, \ldots, n]$.

2) Find the maximum achievable SINR as

$$
\gamma^{*}=\min _{j \in \mathbf{v}} \frac{1}{\lambda^{*}\left(\psi\left(\mathbf{A}, \frac{\boldsymbol{\eta}}{\bar{p}_{j}},\{j\}\right)\right)} .
$$

3) If $\gamma^{*} \geq \gamma_{t h}, \mathbf{v}$ is the set of active users, stop.

4) Update the coefficients $w_{i j}$ as

$$
w_{i j}=\left\{\begin{array}{ll}
\left(\frac{\eta_{j}}{\bar{p}_{j}}\right)^{2}+\sum_{\substack{k=1 \\
k \neq i}}^{m} \sum_{\substack{l=1 \\
l \neq i}}^{m} a_{k l} a_{l k}+2 \sum_{\substack{k=1 \\
k \neq i}}^{m} \frac{\eta_{k}}{\bar{p}_{j}} a_{j k} & j \neq i \\
0 & j=i
\end{array} .\right.
$$

5) Determine the worst link as $\hat{i}=\arg \min _{i} \max _{j} w_{i j}$.

6) Set $\mathcal{R} \leftarrow \mathcal{R} \cup\left\{v_{\hat{i}}\right\}, \mathbf{A} \leftarrow \mathbf{A}^{\hat{i}^{-}}, \mathbf{v} \leftarrow \mathbf{v}^{i}, \overline{\mathbf{i}} \leftarrow \overline{\mathbf{p}}^{\hat{i}^{-}}, \boldsymbol{\eta} \leftarrow \boldsymbol{\eta}^{\hat{i}^{-}}$, and $m \leftarrow m-1$, and go to step 2. 


\section{Case Two: Total Transmit Power Constraint}

When the total power is constrained by $\bar{p}_{t}$, the worst user is determined as

$$
\hat{i}=\arg \max _{i}\left\{\frac{1}{\lambda^{*}\left(\psi^{i^{-}}\left(\mathbf{A}, \frac{\boldsymbol{\eta}}{\bar{p}_{t}},\{1,2, \ldots, n\}\right)\right)}\right\} .
$$

We call this algorithm the Removal Algorithm III-A. Similar to the previous discussions, we propose the following low-complexity algorithm for the user removal with total power constraint (see [25] for details) .

Removal Algorithm III-B

1) Set $\mathbf{A}$ as in (6), $m=n, \mathcal{R}=\varnothing$, and $\mathbf{v}=[1,2, \ldots, n]$.

2) Find the maximum achievable SINR as

$$
\gamma^{*}=\frac{1}{\lambda^{*}\left(\psi\left(\mathbf{A}, \frac{\boldsymbol{\eta}}{\bar{p}_{t}},\{1, \ldots, m\}\right)\right)} .
$$

3) If $\gamma^{*} \geq \gamma_{t h}, \mathbf{v}$ is the set of active users, stop.

4) Update the coefficients $w_{i}$ as

$$
w_{i}=a_{i i}^{2}+2 \sum_{\substack{j=1 \\ j \neq i}}^{m} a_{i j} a_{j i}+2 \frac{\eta_{i}}{\bar{p}_{t}} \sum_{j=1}^{m} a_{j i}+2 \sum_{j=1}^{m} \frac{\eta_{j}}{\bar{p}_{t}} a_{i j}+2 \frac{\eta_{i}}{\bar{p}_{t}} \sum_{\substack{j=1 \\ j \neq i}}^{m} \frac{\eta_{j}}{\bar{p}_{t}}+\left(\frac{\eta_{i}}{\bar{p}_{t}}\right)^{2} .
$$

5) Determine the worst link as $\hat{i}=\arg \max w_{i}$.

6) Set $\mathcal{R} \leftarrow \mathcal{R} \cup\left\{v_{\hat{i}}\right\}, \mathbf{A} \leftarrow \mathbf{A}^{\hat{i}^{-}}, \mathbf{v} \leftarrow \mathbf{v}^{i} \hat{i}^{-}, \boldsymbol{\eta} \leftarrow \boldsymbol{\eta}^{\hat{i}^{-}}, m \leftarrow m-1$, and go to step 2 .

\section{A. Numerical Results}

The simulation results are presented for a Rayleigh fading channel with $n=8$. For the results in a cellular network see [25]. The parameters $g_{i j}$ follow an exponential distribution with mean and variance one for the forward gains, and mean $10^{-2}$ and variance $10^{-4}$ for the cross gains.

We define Outage Probability as the ratio between the number of the inactive users to the total number of the users. This probability shows the percentage of the users that fail to attain the required SINR. We use this function as a metric to compare different algorithms, as it is used in [13], [26].

In [19], a number of removal algorithms when the power of transmitters are individually constrained are proposed. We selected centralized GRN-DCPC to compare it with our results 


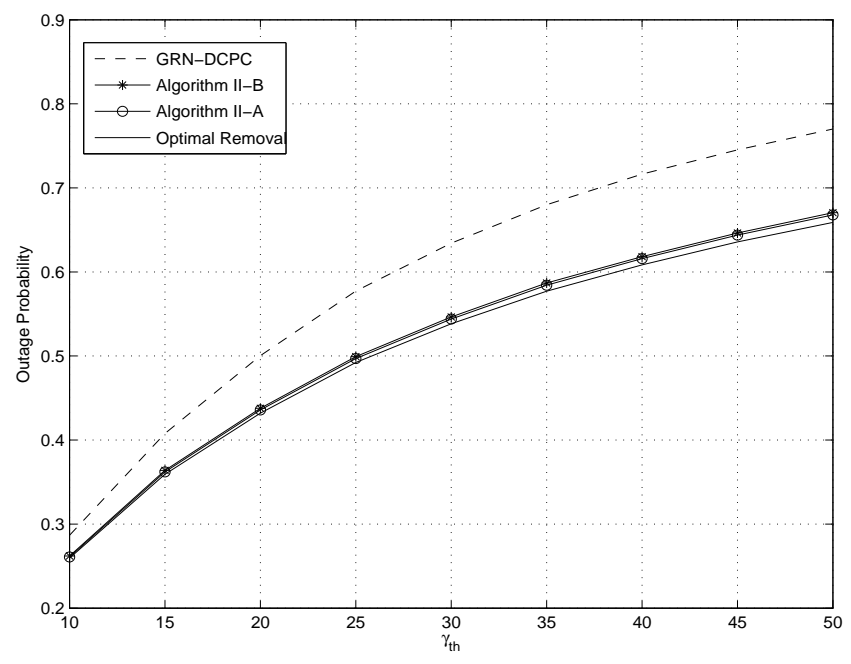

Fig. 5. Constraints on the Power of Individual Transmitters, $\sigma_{i}{ }^{2}=10^{-2}, \bar{p}_{i}=1 \mathrm{w} \forall i$

since according to [19], it outperforms the other presented algorithms in that work. The simulation results in Fig. 5 show a significant improvement in the outage probability of the algorithms II-A and II-B compared to GRN-DCPC. As depicted in Fig. 6, when the total power is bounded, the performance of algorithms III-A and III-B is very close to the optimum result (which is obtained by exhaustive search). To the best of our knowledge, there are no alternative algorithms for the case that the total power is bounded.

\section{CONCLUSION}

In this paper, a communication system including $n$ interfering AWGN links is considered. Each transmitter uses a Gaussian codebook and each receiver only decodes the data of the corresponding transmitter. We have obtained a closed-form solution for the maximum achievable SINR in such a system, utilizing the Perron-Frobenious theorem, when the sum-power on any subset of the users is constrained. This result leads to characterizing the boundary of SINR region for the system. In addition, we consider a time-varying channel where channel gains are selected randomly from a finite set with a certain probability and the average power of the users are constrained linearly. We obtain the zero-outage SINR region for such a system. In addition, we consider the scenario in which the required SINR is out of the SINR region. We develop a user removal algorithm which maximizes the number of users which are active simultaneously 


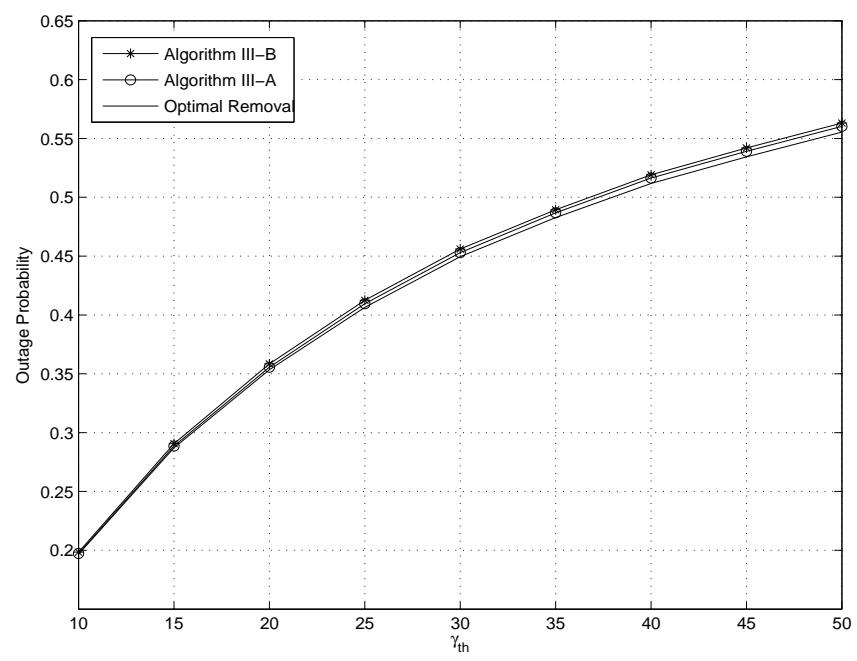

Fig. 6. Constraint on the Total Power, $\sigma_{i}{ }^{2}=10^{-3} \forall i, \bar{p}_{t}=1 \mathrm{w}$

and satisfy the SINR requirement. This algorithm is compared with other alternatives and it is shown that it outperforms the other ones.

\section{APPENDIX}

\section{PROOF OF LEMMA 8}

First, we need the following lemma.

Lemma 12 Let $\mathrm{p}$ and $\hat{\mathrm{p}}$ be two power vectors with the corresponding SINR vectors $\gamma$ and $\hat{\gamma}$, respectively. Consider the non-empty index set $\mathcal{I} \subseteq\{1, \cdots, n\}$. Assume the power vectors satisfy

$$
\begin{array}{ll}
\hat{p}_{i}=\frac{1}{\alpha} p_{i}, & \forall i \in \mathcal{I} \\
\hat{p}_{i} \geq p_{i}, & \forall i \in \mathcal{I}^{c},
\end{array}
$$

where $\alpha>1$ is a constant and $\mathcal{I}^{c}=\{1, \cdots, n\} \backslash \mathcal{I}$. Then, we have

$$
\hat{\gamma}_{i}<\gamma_{i} \quad \forall i \in \mathcal{I}
$$

It is noteworthy that the special case of $\mathcal{I}=\{1, \cdots, n\}$ corresponds to the case that $\hat{\mathbf{p}}=\frac{1}{\alpha} \mathbf{p}$. In this case, we have $\hat{\gamma}<\gamma$. 
Proof: From the definition of SINR in (1), for any $i \in \mathcal{I}$, we have

$$
\begin{aligned}
\hat{\gamma}_{i} & =\frac{g_{i i} \hat{p}_{i}}{\sigma_{i}^{2}+\sum_{\substack{j=1 \\
j \neq i}}^{n} g_{i j} \hat{p}_{j}} \\
& =\frac{g_{i i} \hat{p}_{i}}{\sigma_{i}^{2}+\sum_{j \in \mathcal{I}^{c}} g_{i j} \hat{p}_{j}+\sum_{\substack{j \in \mathcal{I} \\
j \neq i}} g_{i j} \hat{p}_{j}} \\
& \stackrel{\frac{1}{\alpha} g_{i i} p_{i}}{\leq} \frac{g_{i i} p_{i}}{\sigma_{i}^{2}+\sum_{j \in \mathcal{I}^{c}} g_{i j} p_{j}+\frac{1}{\alpha} \sum_{\substack{j \in \mathcal{I} \\
j \neq i}} g_{i j} p_{j}} \\
& <\frac{\sigma_{i}^{2}+\sum_{j \in \mathcal{I}^{c}} g_{i j} p_{j}+\sum_{\substack{j \in i \\
j \neq i}} g_{i j} p_{j}}{\sigma_{i},}
\end{aligned}
$$

where the last inequality is because $\alpha>1$ and the right-hand-side of (41) is a decreasing function of $\alpha$.

Now, we are ready to prove Lemma 8 . Given that $\gamma \leq \hat{\gamma}$, we should prove that the index set $\mathcal{J}=\left\{i: 1 \leq i \leq n, \hat{p}_{i}<p_{i}\right\}$ is empty. To this end, we show that if $\mathcal{J}$ is not empty then, there exists some $i \in \mathcal{J}$ for which $\hat{\gamma}_{i}<\gamma_{i}$; this is a contradiction to $\gamma \leq \hat{\gamma}$.

For the special case that $\mathbf{p}$ and $\hat{\mathbf{p}}$ satisfy condition (39) (with $\mathcal{I}=\mathcal{J}$ ), Lemma 12 can be applied and it is concluded that $\hat{\gamma}_{i}<\gamma_{i}$ for all $i \in \mathcal{J}$. This completes the proof.

For the case that condition (39) is not satisfied, we prove the claim by induction on $|\mathcal{J}|$ (cardinality of $\mathcal{J}$ ).

For $|\mathcal{J}|=1$, only one component, say $i$, of $\hat{\mathbf{p}}$ is less than the corresponding component in $\mathbf{p}$. Thus, we have $\hat{\gamma}_{i}<\gamma_{i}$. Now, we should show that if our claim is true for any $|\mathcal{J}| \in\{1, \cdots, \ell\}$, i.e., if

$$
1 \leq|\mathcal{J}| \leq \ell \quad \Rightarrow \quad \exists i \in \mathcal{J}: \quad \hat{\gamma}_{i}<\gamma_{i}
$$

then, it is true for $|\mathcal{J}|=\ell+1$ as well, i.e.,

$$
|\mathcal{J}|=\ell+1 \quad \Rightarrow \quad \exists i \in \mathcal{J}: \quad \hat{\gamma}_{i}<\gamma_{i}
$$

Let us define

$$
\alpha^{*}=\min _{i \in \mathcal{J}}\left\{\frac{p_{i}}{\hat{p}_{i}}\right\}
$$

and choose a new power vector $\mathbf{p}^{*}=\alpha^{*} \hat{\mathbf{p}}$ with the corresponding SINR vector $\gamma^{*}$. According to the definition of $\mathbf{p}^{*}$, for the corresponding index set $\mathcal{J}^{*}=\left\{j: 1 \leq j \leq n, p_{j}^{*}<p_{j}\right\}$ it is clear 
that $\left|\mathcal{J}^{*}\right|<|\mathcal{J}|$, which means the cardinality of $\mathcal{J}^{*}$ is at most equal to $\ell$. Also, since $\mathbf{p}$ and $\hat{\mathbf{p}}$ do not satisfy condition (39), $\mathcal{J}^{*}$ is not empty. By utilizing the assumption of induction it is concluded that there exists some $j \in \mathcal{J}^{*}$ for which $\gamma_{j}^{*}<\gamma_{j}$. On the other hand, since $\alpha^{*}>1$, $\mathbf{p}^{*}$ and $\hat{\mathbf{p}}$ satisfy condition (39) with $\mathcal{I}=\{1, \cdots, n\}$. According to Lemma $12, \hat{\gamma}_{j}<\gamma_{j}^{*}$ for all $j \in\{1, \cdots, n\}$. Hence, it is concluded that $\hat{\gamma}_{j}<\gamma_{j}$ for some $j \in \mathcal{J}^{*}$ and the proof is complete.

\section{ACKNOWLEDGMENT}

The authors would like to thank Mohammad A. Maddah-Ali for helpful discussions.

\section{REFERENCES}

[1] R. D. Yates, "A framework for uplink power control in cellular radio systems," IEEE Journal on Selected Areas in Communications, vol. 13, no. 7, pp. 1341 - 1347, 1995.

[2] D. Catrein, L. A. Imhof, and R. Mathar, "Power control, capacity, and duality of uplink and downlink in cellular CDMA systems," IEEE Transactions on Communications, vol. 52, no. 10, pp. 1777 - 1785, October 2004.

[3] L. A. Imhof and R. Mathar, "Capacity regions and optimal power allocation for CDMA cellular radio," IEEE Transactions on Information Theory, vol. 51, no. 6, pp. 2011 - 2019, June 2005.

[4] H. Boche and S. Stanczak, "Convexity of some feasible QoS regions and asymptotic behavior of the minimum total power in CDMA systems," IEEE Transactions on Communications, vol. 52, no. 12, pp. 2190 - 2197, December 2004.

[5] — "Log-convexity of the minimum total power in CDMA systems with certain quality-of-service guaranteed," IEEE Transactions on Information Theory, vol. 51, no. 1, pp. 374 - 381, January 2005.

[6] C. W. Sung, "Log-convexity property of the feasible SIR region in power-controlled cellular systems," IEEE Communications Letters, vol. 6, no. 6, pp. 248 - 249, June 2002.

[7] S. Stanczak and H. Boche, "The infeasible SIR region is not a convex set," IEEE Transactions on Communications, vol. 54, no. 11 , pp. 1905 - 1907, November 2006.

[8] R. Etkin, A. Parekh, and D. N. C. Tse, "Spectrum sharing for unlicensed bands," in First IEEE International Symposium on New Frontiers in Dynamic Spectrum Access Networks, November 2005, pp. 338 - 345.

[9] S. G. Kiani and D. Gesbert, "Maximizing the capacity of large wireless networks: Optimal and distributed solutions," in IEEE International Symposium on Information Theory, July 2006, pp. 2501 - 2505.

[10] L. Xiao, M. Johansson, and S. P. Boyd, "Simultaneous routing and resource allocation via dual decomposition," IEEE Transactions on Communications, vol. 52, no. 7, pp. 1136 - 1144, July 2004.

[11] S. V. Hanly, "An algorithm for combined cell-site selection and power control to maximize cellular spread spectrum capacity," IEEE Journal on Selected Areas in Communications, vol. 13, no. 7, pp. 1332 - 1340, September 1995.

[12] J. M. Aein, "Power balancing in systems employing frequency reuse," in Comsat Tech. Rev., vol. 3, 1973.

[13] J. Zander, "Performance of optimum transmitter power control in cellular radio systems," IEEE Transactions on Vehicular Technology, vol. 41, no. 1, pp. 57 - 62, February 1992.

[14] D. N. C. Tse and S. V. Hanly, "Linear multiuser receivers: effective interference, effective bandwidth and user capacity," Automatica, vol. 35, no. 12, pp. 1987 - 2012, March 1999. 
[15] H. Boche and S. Stanczak, "Iterative algorithm for finding optimal resource allocations in symbol-asynchronous CDMA channels with different SIR requirements," in Thirty-Sixth Asilomar Conference on Signals, Systems and Computers, vol. 2, no. 3-6, November 2002, pp. 1909 - 1913.

[16] A. J. Goldsmith and P. P. Varaiya, "Capacity of fading channels with channel side information," IEEE Transactions on Information Theory, vol. 43, no. 6, pp. 1986 - 1992, November 1997.

[17] A. J. Goldsmith, Wireless Communications. Cambridge University Press, 2004.

[18] N. Jindal, J. G. Andrews, and S. Weber, "Bandwidth-SINR tradeoffs in spatial networks," in International Symposium on Information Theory, June 2007.

[19] M. Andersin, Z. Rosberg, and J. Zander, "Gradual removals in cellular PCS with constrained power control and noise," Wireless Networks, vol. 2, no. 1, pp. 27 - 43, January 1996.

[20] J. Lin, T. Lee, and Y. Su, "Downlink power control algorithms for cellular radio systems," IEEE Transactions on Vehicular Technology, vol. 44, no. 1, February 1995.

[21] E. Seneta, Non-Negative Matrices: An Introduction to Theory and Applications. John Wiley and Sons, January 1973.

[22] M. Chiang, "Geometric programming for communication systems," Foundations and Trends of Communications and Information Theory, vol. 2, no. 1-2, pp. 1-156, August 2005.

[23] D. Julian, M. Chiang, D. O’Neill, and S. Boyd, "QoS and fairness constrained convex optimization of resource allocation for wireless cellular and ad hoc networks," in IEEE Infocom, vol. 2, June 2002, pp. 477-486.

[24] R. G. Horn and C. A. Johnson, Matrix Analysis. Cambridge University Press, 1985.

[25] H. Mahdavi-Doost, "Characterization of rate region and user removal in interference channels with constrained power," Master's thesis, University of Waterloo, 2007, available at www.cst.uwaterloo.ca.

[26] J. Zander, "Distributed cochannel interference control in cellular radio systems," IEEE Transactions on Vehicular Technology, vol. 41, no. 3, pp. 305 - 311, August 1992. 